\title{
Multiphoton autofluorescence lifetime imaging of induced pluripotent stem cells
}

Aisada Uchugonova 


\title{
Multiphoton autofluorescence lifetime imaging of induced pluripotent stem cells
}

\author{
Aisada Uchugonova a,b,* \\ aSaarland University, Department of Biophotonics and Laser Technology, Saarbrücken, Germany \\ bJenLab GmbH, Jena, Germany
}

\begin{abstract}
The multiphoton fluorescence lifetime imaging tomograph MPTflex with its flexible 360-deg scan head, articulated arm, and tunable femtosecond laser source was employed to study induced pluripotent stem cell (iPS) cultures. Autofluorescence (AF) lifetime imaging was performed with 250-ps temporal resolution and submicron spatial resolution using time-correlated single-photon counting. The two-photon excited AF was based on the metabolic coenzymes $\mathrm{NAD}(\mathrm{P}) \mathrm{H}$ and flavin adenine dinucleotide/flavoproteins. iPS cells generated from mouse embryonic fibroblasts (MEFs) and cocultured with growth-arrested MEFs as feeder cells have been studied. Significant differences on AF lifetime signatures were identified between iPS and feeder cells as well as between their differentiating counterparts. ๑ 2017 Society of Photo-Optical Instrumentation Engineers (SPIE) [DOI: 10.1117/1.JBO.22.6 .066018]
\end{abstract}

Key words: induced pluripotent stem cells; feeder cells; two-photon microscopy; multiphoton tomography; fluorescence lifetime imaging; autofluorescence.

Paper 170125PRR received Feb. 23, 2017; accepted for publication Jun. 7, 2017; published online Jun. 30, 2017.

\section{Introduction}

Artificially generated pluripotent stem cells, known as induced pluripotent stem cells (iPS cells), resemble embryonic stem (ES) cells. Therefore, they are of high interest especially in regenerative medicine. By contrast, most of the tissue-specific stem cells found within the majority of the organs are limited in their differentiation ability. ${ }^{1,2}$ ES cells are pluripotent and can generate any type of tissue-specific functional cells. Applications of ES cells are however limited due to the varying immunogenetic backgrounds of patients as well as ethically controversial debates on the usage of embryos for research. ${ }^{3,4}$ Yamanaka and Takahashi ${ }^{5}$ discovered iPS cells in 2006, as an alternative to ES cells. They found that fully differentiated somatic cells can be reprogrammed into an ES cell-like state and received the Nobel Prize in 2012 for this discovery. ${ }^{5,6}$ In their study, the introduction of the transcription factors Oct4, Sox2, c-Myc, and Klf4 stimulated fibroblasts into becoming iPS cells. This breakthrough result of Yamanaka and Takahashi ${ }^{5}$ has taken stem cell research to the next level, where generated iPS cells can be unrestrictedly used to produce functional cells in addition to the advantages of their immunologenic coherence to the patients.

The typical iPS culture system is maintained on growtharrested mouse embryonic fibroblasts (MEFs) known as feeder cells. Feeder-free methods may cause cell genetic instability and offer a poor cell attachment. ${ }^{7}$ So far, fluorescence/magnetic labeling is utilized to monitor cellular behavior in such heterogeneous coculture systems in order to differentiate between different cell types and for cell separation purposes (removal of feeder cells). However, separation and characterization remain time consuming and problematic because exogenous labels are employed.
An efficient and label-free approach is highly desired for rapid assessment of complex iPS cultures and essential for clinical applications of iPS cells.

In multiphoton microscopy, near-infrared (NIR) picojoule femtosecond laser pulses are used. ${ }^{8}$ By using multiphoton microscopy, photodestruction and impact on cellular metabolism can be avoided. Noninvasive imaging of live cells can be realized due to the subfemtoliter absorption volume of NIR, using high numerical aperture (NA) objectives. Multiphoton excitation is suitable for the excitation of natural fluorophores [autofluorescence (AF)] with an absorption maximum within the UV/blue range, such as nicotinamide adenine dinucleotide $[\mathrm{NAD}(\mathrm{P}) \mathrm{H}]$ and flavin adenine dinucleotide (FAD)/flavoproteins. ${ }^{9}$ Those fluorophores are important metabolic coenzymes that are involved in cellular respiration processes as they provide information on metabolic changes during cell growth and differentiation. Therefore, optical metabolic imaging can be performed based on AF detection.

Fluorescence lifetime imaging (FLIM) is a convenient method to quantify the relative concentration of bound and free $\mathrm{NAD}(\mathrm{P}) \mathrm{H}$ as well as flavins by utilizing the varying fluorescence lifetimes without affecting the integrity of the cell structure. ${ }^{10,11}$ Current studies have demonstrated that energy metabolism plays a critical role in stem cells maintenance and differentiation. ${ }^{12}$ Recently, multiphoton-FLIM was used to investigate stem cells during differentiation based on AF. Shifts in AF lifetimes of NAD $(\mathrm{P}) \mathrm{H}$ and bound/free NAD $(\mathrm{P}) \mathrm{H}$ ratios during stem cell differentiation into mature cells have been reported. ${ }^{13-17}$ Such metabolic changes indicate the reconfiguration of metabolic states from glycolysis into phosphorylated oxidation during differentiation.

The goal of this multiphoton AF lifetime imaging study was to investigate optically the metabolic coenzymes of highly 
proliferative iPS cells in comparison to cocultured nonproliferative MEFs. Unlike conventional methods, multiphoton time-correlated AF detection is completely noninvasive and label-free and is a "pin-point" method for metabolic validation of clinically relevant iPS cells.

\section{Materials and Methods}

\subsection{Cell Cultures}

Mouse iPS cells originated from MEFs and growth-arrested MEFs were used for this study. Mouse primary iPS cells were generated from MEFs through infection with Oct4, Sox2, Klf4, and c-Myc-expressing retroviral vectors (Stemgent \#08-0007). They were cultured under standard mouse embryonic cell culture conditions. Culture dishes with $170-\mu \mathrm{m}$ glass cover slips and with 500 grids (Ibidi \#81168) were used for iPS colony tracking before FLIM imaging and after immunolabeling. IPS medium consisting of KO-DMEM medium (\#10829, Gibco), 20\% ES-qualified FBS (\#16141, Gibco), 0.1 mM MEM NEAA (nonessential amino acids) (100×, \#11140-50, Gibco), $0.1 \mathrm{mM}$ mercapthoethanol (\#M7522, Sigma), as well as 8-ng/ml beta-FGF (\#CF003, Millipore) were used. Growtharrested MEFs were used as feeder cells (\#GSC-6001G, GlobalStem). Feeder cells were prepared as monolayer in ES cells qualified Dulbecco medium (ES-DMEM) (\#GSM-2001, Global-Stem) and 15\% FBS (\#GSM-6002, Global-Stem) 1 day prior passaging iPS cells. Feeder cells were seeded in a density of $5 \times 10^{4}$ to $10 \times 10^{4}$ cells $/ \mathrm{cm}^{2}$. Cells were washed with warm Dulbecco's phosphate-buffered saline and the medium was exchanged to iPS cell medium up to $2 \mathrm{~h}$ before transferring iPS cells. IPS colony formation was monitored using a standard microscope (Zeiss AxioObserver). The entire culture procedure is illustrated in Fig. 1. The morphology of mouse iPS cells and capability to form 3-D colonies was identical to that of mouse ES cells. By contrast, MEFs (referred as feeder cells) have an elongated morphology and grow as a monolayer in the culture dish.

\subsection{Immunocytochemistry}

After FLIM measurements, the undifferentiated state of iPS cells was confirmed by the expression of the pluripotency live staining marker SSEA1. Live cell colonies were stained with the fluorescent antibody StainAlive DyLight Cy3 SSEA1 (Stemgent \#130-099-035) in a sterile working environment. The primary antibody was diluted to a final concentration of $2.5 \mu \mathrm{g} / \mathrm{ml}$ in fresh cell culture medium. The medium was aspirated from the cells and the diluted primary antibody was given to the live cells. Cells were incubated for $30 \mathrm{~min}$ at $37^{\circ} \mathrm{C}$ and $5 \%$ $\mathrm{CO}_{2}$. Afterward, the primary antibody was washed gently two times with the cell culture medium and cells were examined under the fluorescence microscope with LED module (Zeiss AxioObserver). The excitation filter BP550/25, the beam splitter FT570, and the emission filter BP $605 / 70$ were used for the detection of Cy3 SSEA1.

\subsection{Multiphoton Autofluorescence Lifetime Imaging}

The FLIM-MPTflex tomograph (JenLab GmbH) equipped with a NIR-fs tunable laser emitting in the spectral range of 720 to $920 \mathrm{~nm}$ was used to perform multiphoton AF lifetime measurements (Fig. 2). The filters $680 \mathrm{SP}$ and $409 \mathrm{LP}$ were employed to detect the AF between 409 and $680 \mathrm{~nm}$. 3-D two-photon AF images were acquired in $512 \times 512$ pixel fields at 14 -s scan time and by optical sectioning with $5-\mu \mathrm{m} \mathrm{z}$-steps. Fluorescence emission was recorded through the focusing optics (NA 1.3). The mean power was adjusted at $5 \mathrm{~mW}$. AF lifetime imaging was

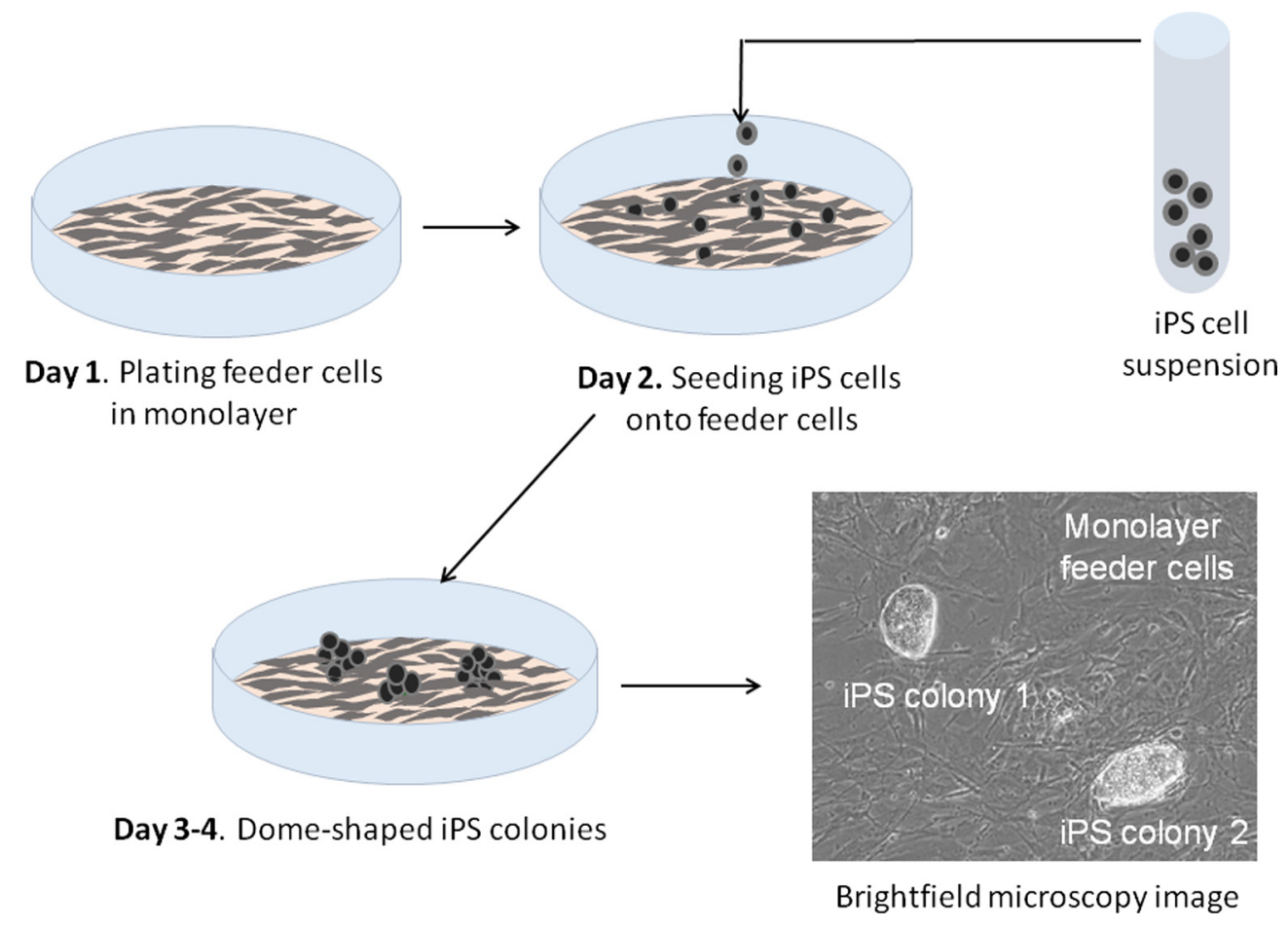

Fig. 1 Graphical representation of the iPS culture method onto feeder cells. Feeder cells have an elongated morphology and grow as a monolayer. IPS cells seeded onto feeder cells have a morphology similar to mouse ES cells and form 3-D colonies. 


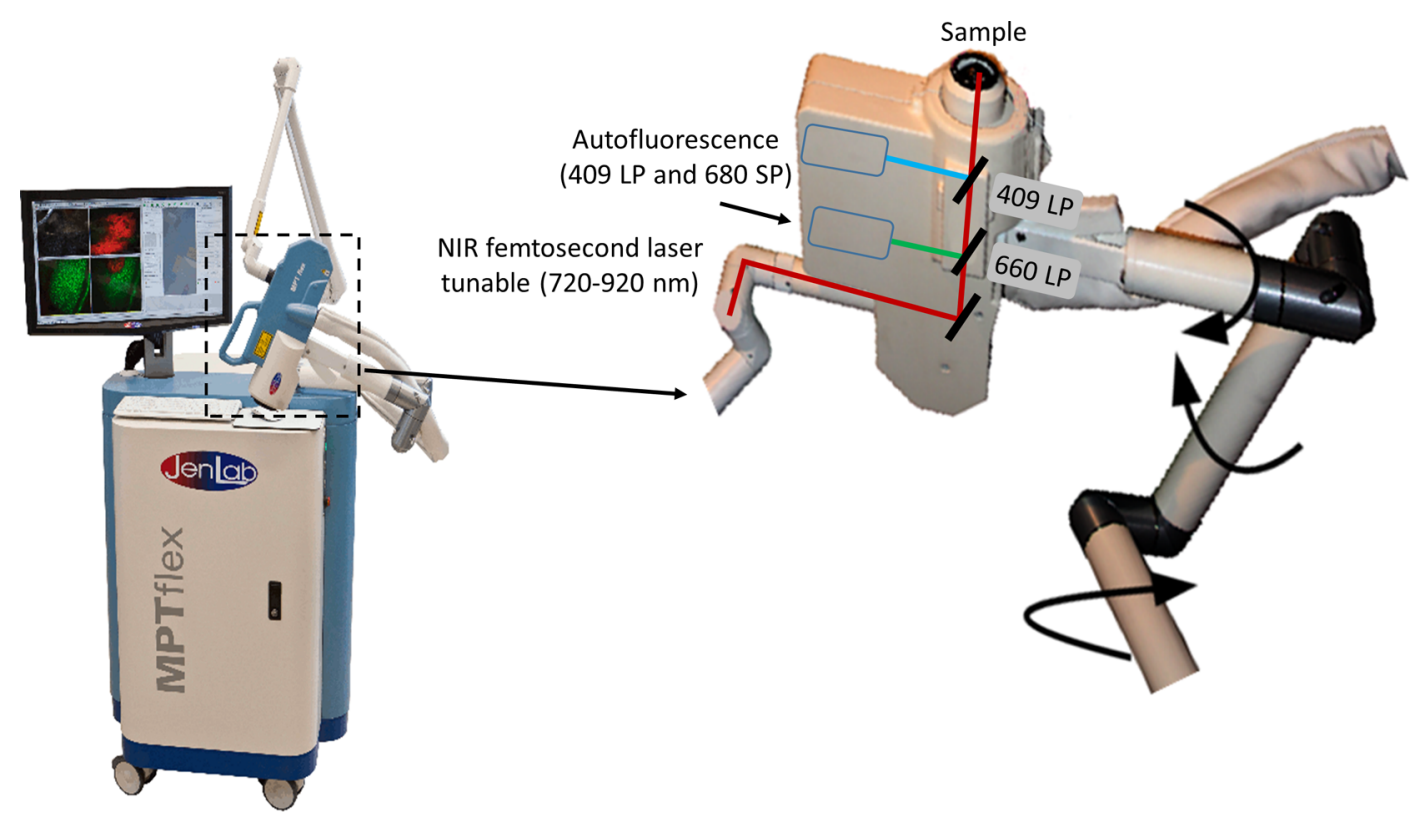

Fig. 2 FLIM-MPTflex tomograph with 360-deg scan head containing an AF detection unit used for the multiphoton AF lifetime imaging. LP, longpass filter; SP, shortpass filter; NIR, near-infrared.

based on time-correlated single-photon counting with a fast photomultiplier detector PMC-100. For the analyses of the obtained fluorescence decay curves per pixel, the SPCImage software (Becker\&Hickl GmbH) was used. The nonlinear least square curve fitting method was employed for the analysis of two fluorescence components per pixel that represent molecules of free and bound form of NAD(P)H and FAD/flavoproteins. Because there is only one detector for AF detection in the current MPT system available, we have chosen two excitation wavelengths 740 and $850 \mathrm{~nm}$ in order to separate fluorescence of NAD(P) $\mathrm{H}$ from $\mathrm{FAD} /$ flavoproteins. Indeed, there is an overlap of NAD(P)H and FAD two-photon excitation spectra and we excite both fluorophores when using the 740-nm laser wavelength. ${ }^{18,19}$ Based on the two-photon excitation cross-section, ${ }^{18}$ FAD/flavoproteins can be selectively excited at $850 \mathrm{~nm}$ without the excitation overlap of $\mathrm{NAD}(\mathrm{P}) \mathrm{H}$. However, the NAD $(\mathrm{P}) \mathrm{H}$ signal with a fluorescence maximum in the range of 440 to $460 \mathrm{~nm}$ seems to be the more prominent signal in many cells and tissues when using laser wavelength beneath $800 \mathrm{~nm} .{ }^{19}$ This might be due to the relatively low cellular concentration of flavin and flavoproteins compared with $\mathrm{NAD}(\mathrm{P}) \mathrm{H}^{20,21}$

Two fluorescence lifetime components $\tau_{1}$ and $\tau_{2}$ were extracted (biexponential fitting) along with their relative contributions given as $\alpha_{1}$ and $\alpha_{2}$, respectively $\left(\alpha_{1}+\alpha_{2}=1\right)$. Additionally, a mean fluorescence lifetime $\left(\tau_{\mathrm{m}}\right)$ value per pixel was calculated and depicted $\left(\tau_{\mathrm{m}}=\alpha_{1} \tau_{1}+\alpha_{2} \tau_{2}\right)$ as false-color coded FLIM image. The goodness of fits of the results $\left(\chi^{2}\right)$ lies between 0.9 and 1.3 (close to the ideal value 1) across the images. A triple exponential fit was also investigated and yielded nearly the same $\chi^{2}$ and mean values $\left(\tau_{\mathrm{m}}\right)$ as the biexponential fit (but with a larger variation in $\tau_{1}, \tau_{2}$, and $\tau_{3}$ ). Therefore, the biexponential fit was used to indicate lifetime values of iPS and feeder cells. The instrument response function was measured using the SHG signal from collagen. A full width of half maximum (FWHM) value of $\sim 250$ ps was determined. Measurements were performed in a fluorescence-free medium under room temperature.

\subsection{Statistical Analysis}

For statistical analysis, 10 iPS colonies (total number of cells about 500) and several regions of interest areas covering feeder cells (total number of cells about 100) were used. Data are represented as average values \pm standard deviation. A two-tailed test using student's $t$-test was carried out to determine significant differences in the mean values between iPS colonies and feeder cells. $P<0.05$ value signifies significance. Statistical analysis was performed using MS Excel for MacOS X and Origin Version 9.0.

\section{Results}

IPS colonies and feeder cells within the same culture dishes were imaged by their intrinsic AF without the usage of any external fluorophores. Highly compact 3-D colonies with a diameter of 50 to $100 \mu \mathrm{m}$ and with a morphology pattern similar to mouse ES cell colonies were chosen for multiphoton AF lifetime imaging. Two-photon excitation allowed subsequent optical imaging at multiple depths of the entire iPS colony. Spatially resolved autofluorescence decay curves of iPS cells at the excitation wavelengths of 740 and $850 \mathrm{~nm}$ have been obtained.

Figure 3 represents one optical autofluorescence section out of z-stacks of an iPS colony containing about 50 cells in total. An intensity and a false-color coded FLIM image of the same iPS colony is demonstrated. The color gradients of the FLIM image from red (500 ps) to blue (1200 ps) represent additional information on the temporal behavior of the AF.

When analyzing 10 colonies excited at $740 \mathrm{~nm}$ [mainly NAD (P)H is excited], $\tau_{2}$ values are distributed between 1000 and 3000 ps with an average peak of $(2181 \pm 77)$ ps. The mean values $\left(\tau_{\mathrm{m}}\right)$ are between 500 and 1200 ps with a peak of $(802 \pm$ 22) ps and the $\tau_{1}$ values between 300 and 700 ps with a peak of $(464 \pm 31)$ ps. At 850 -nm excitation wavelength, where no $\mathrm{NAD}(\mathrm{P}) \mathrm{H}$ can be excited but flavins, $\tau_{2}$ values had an average peak of $(1738 \pm 57)$ ps. The mean values $\left(\tau_{\mathrm{m}}\right)$ had a peak of $(626 \pm 48)$ ps and $\tau_{1}$ values with a peak of $(312 \pm 36)$ ps. 

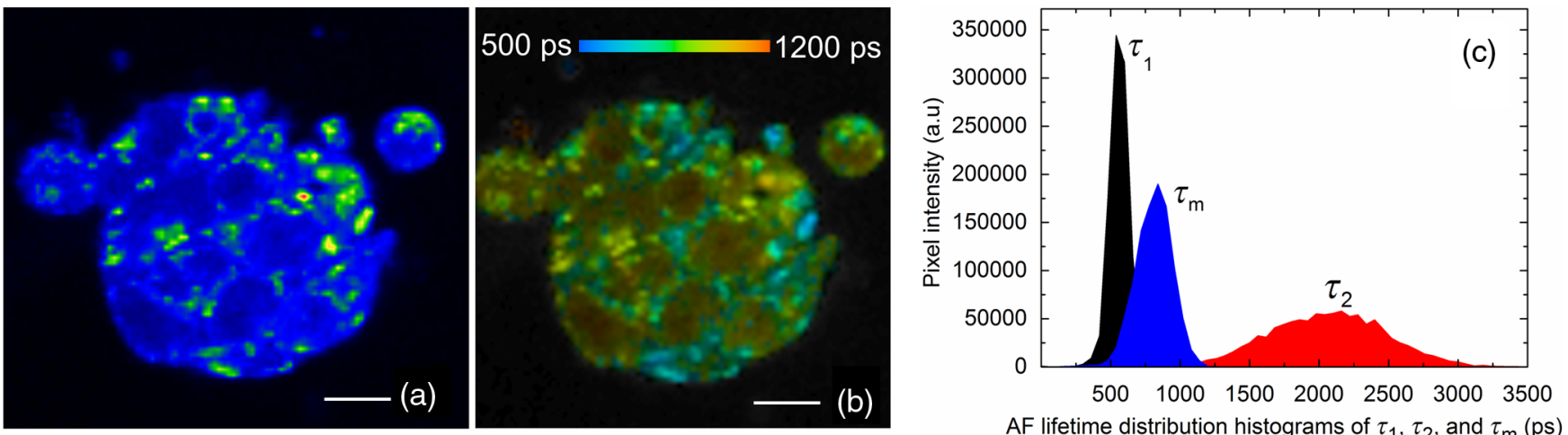

Fig. 3 Representative intensity and AF lifetime optical section. (a) Intensity and (b) false-color coded AF lifetime image of the mean $\mathrm{AF}$ lifetime value $\left(\tau_{\mathrm{m}}\right)\left(\tau_{\mathrm{m}}=\alpha_{1} \tau_{1}+\alpha_{2} \tau_{2}\right)$ between 500 and $1200 \mathrm{ps}$ using biexponential fitting (740-nm excitation). Scale bar: $10 \mu \mathrm{m}$. (c) The short $\left(\tau_{1}\right)$, mean $\left(\tau_{\mathrm{m}}\right)$, and long $\left(\tau_{2}\right)$ lifetime distribution histograms are shown.

In a next step, the AF lifetime values of feeder cells were compared with iPS cells. As shown in Fig. 4, iPS cells have significantly longer $\left(\tau_{\mathrm{m}}\right)$ lifetimes than feeder cells. Further AF lifetime values $\left(\tau_{1}, \tau_{\mathrm{m}}\right.$, and $\left.\tau_{2}\right)$ of iPS and feeder cells when excited at 740 and $850 \mathrm{~nm}$ are given in Table 1 .

The range and overall distribution of those fluorescence lifetime values for both iPS and feeder cells can be visualized in Box-Whisker plots depicted in Fig. 5. The middle line dividing the box into two parts shows the median of the data. The middle "box" represents the middle $50 \%$ of data and fall within the interquartile range. The upper and lower whiskers (maximum and minimum values, respectively) represent data outside the middle $50 \%$. The significant difference between iPS cells and feeder cells is clearly seen $(40 \%$ higher mean AF lifetime of iPS versus feeder cells).

\subsection{Autofluorescence Lifetime Imaging of Differentiating iPS Colonies}

Some iPS colonies did not express the pluripotency marker SSEA1 most likely due to spontaneous differentiation. These iPS cell colonies were also investigated. Interestingly, differentiating
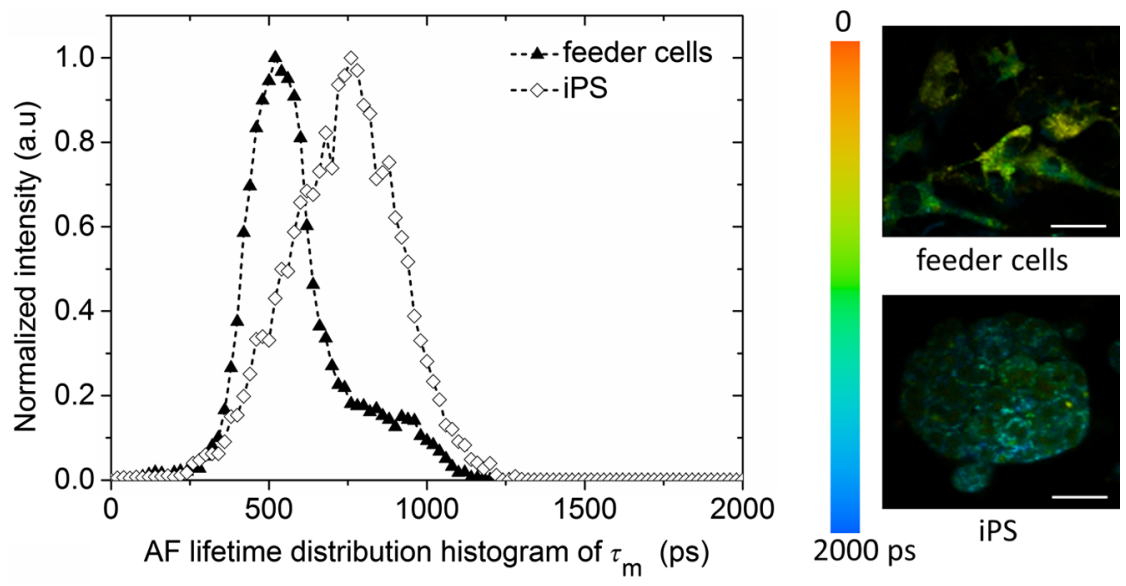

Fig. 4 Mean $\left(\tau_{\mathrm{m}}\right)$ AF lifetime histogram and false-color coded AF lifetime image of feeder cells versus iPS. $\tau_{\mathrm{m}}$ displays between 0 and $2000 \mathrm{ps}$ (red to blue) using biexponential fitting (740-nm excitation). Scale bar: $10 \mu \mathrm{m}$. IPS cells have significantly longer $\left(\tau_{\mathrm{m}}\right)$ lifetime than feeder cells.

Table 1 Mean AF lifetimes at different excitation wavelengths. Free and protein-bound NAD(P)H is excited at $740 \mathrm{~nm}$, whereas FAD/flavoproteins are excited at $850 \mathrm{~nm}$. The average data and standard deviation (SD) were calculated from $10 \mathrm{iPS}$ colonies (containing 500 cells) and from feeder cells (about 100 cells).

\begin{tabular}{lccccccc} 
& \multicolumn{3}{c}{$\mathrm{NAD}(\mathrm{P}) \mathrm{H}$} & & \multicolumn{3}{c}{ FAD/flavoproteins } \\
\cline { 2 - 3 } & $\tau_{\mathrm{m}} \pm \mathrm{SD}(\mathrm{ps})$ & $\tau_{1} \pm \mathrm{SD}(\mathrm{ps})$ & $\tau_{2} \pm \mathrm{SD}(\mathrm{ps})$ & & $\tau_{\mathrm{m}} \pm \mathrm{SD}(\mathrm{ps})$ & $\tau_{2} \pm \mathrm{SD}(\mathrm{ps})$ & $\tau_{1} \pm \mathrm{SD}(\mathrm{ps})$ \\
\hline iPS & $802 \pm 22$ & $464 \pm 31$ & $2181 \pm 77$ & & $626 \pm 48$ & $312 \pm 36$ & $1738 \pm 57$ \\
Feeder cells & $574 \pm 71$ & $287 \pm 137$ & $1595 \pm 41$ & & $503 \pm 19$ & $247 \pm 23$ & $1571 \pm 153$ \\
\hline
\end{tabular}



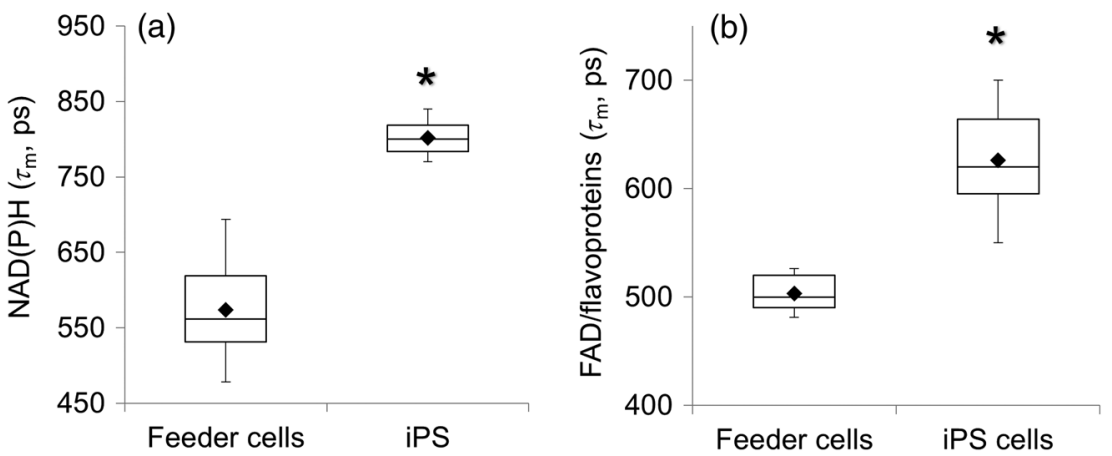

Fig. 5 Feeder cells have significantly shorter mean AF lifetime values in comparison to iPS cells as demonstrated in the Box-Whisker plot. Excitation wavelengths (a) $740 \mathrm{~nm}(\mathrm{NAD}(\mathrm{P}) \mathrm{H})$ and (b) $850 \mathrm{~nm}$ (FAD/flavoproteins). The rhombi denote the average lifetime and asterisks denote significance between iPS and feeder cell values $(p<0.05)$.
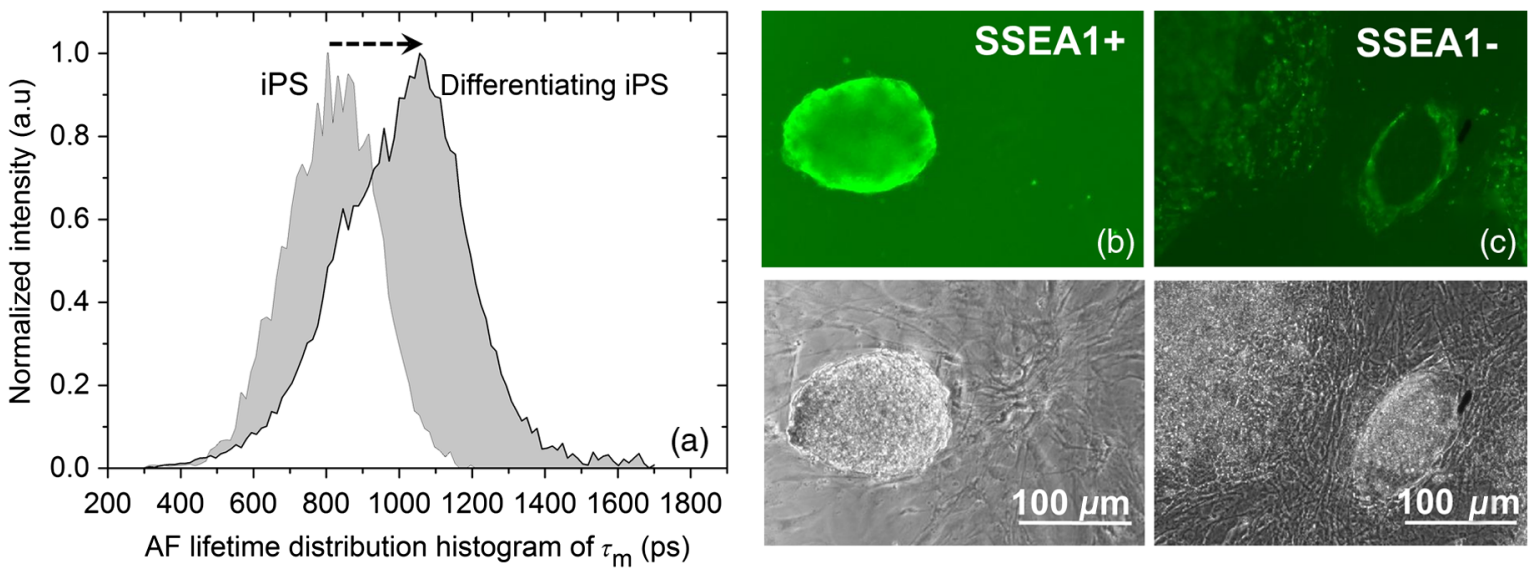

Fig. $6 \mathrm{AF}$ mean lifetime $\tau_{\mathrm{m}}$ : histograms of nondifferentiating and spontaneous differentiating iPS cell colonies. (a) AF lifetime distribution histogram of SSEA1+ expressing iPS colonies have a smaller FHWM $\tau_{\mathrm{m}}$ histogram and shorter $t_{\mathrm{m}}$ values compared to non-SSEA1 expressing iPS cell colonies; (b) fluorescence and brightfield images of an iPS colony expressing the pluripotency marker SSEA1+; (c) fluorescence and brightfield images of an IPS colony with negative SSEA1- signal (spontaneous differentiation).

iPS colonies (SSEA1-) were found to have a significantly longer mean AF lifetime decay and a larger FWHM value of the $t_{\mathrm{m}}$-histogram (Fig. 6) in comparison to SSEA1+ expressing iPS colonies.

\section{Discussion and Conclusion}

Previously, our research group demonstrated the advantages of label-free imaging of stem cells based on multiphoton AF and second harmonic generation microscopy. ${ }^{22}$ Two-photon AF FLIM microscopy was employed to assess metabolic reorganisations in stem cells undergoing osteogenic, adipogenic, and chondrogenic differentiation. ${ }^{13-17,22,23}$ Stringary et al. ${ }^{14}$ applied time-resolved AF using the phasor approach to identify proliferative intestinal stem cells within the live murine small intestine. Endogenous fluorophores were used in order to assess metabolic responses of iPS cell-derived cardiomyocytes to detect drug-induced stress. Large shift toward free $\mathrm{NAD}(\mathrm{P}) \mathrm{H}$ indicated a rise of free/bound NADH ratio. ${ }^{24}$

Within this study, for the first time, AF lifetime values of highly proliferative iPS cells were investigated under physiological conditions and in a three-dimensional environment by noninvasive multiphoton FLIM tomography and compared with cocultured nonproliferative growth-arrested MEFs. Interestingly, significant differences in AF lifetime values were detected. IPS cells had a nearly 1.4-fold higher mean AF lifetime. Some evidence suggested that highly proliferative cells, such as iPS and ES cells, have higher lactate production and oxygen consumption rates, which rely mostly on glycolysis rather than oxidative phosphorylation to rapidly fulfill their energy demand. ${ }^{25}$ These various metabolic characteristics may explain the significant differences in lifetime between highly proliferative iPS cells and nonproliferative feeder cells.

In accordance to previous reports, our results also highlight AF lifetime shifts in their differentiating counterparts. Spontaneous differentiating iPS cells (SSEA1-) had higher mean value.

Such metabolic shifts between nondifferentiated into differentiated cells was also reported earlier when studying adult stem and progenitor cells. ${ }^{13,14}$ Reversed trends in fluorescence lifetimes were detected in spontaneous differentiating murine and human ES cells. ${ }^{26,27}$ Further studies are required to better understand the changes in autofluorescence lifetime signatures of pluripotent stem cells and the details of metabolic reorganization induced by differentiation. In fact, a variety of studies have 
identified metabolic switch from glycolysis to an oxidative state upon differentiation. ${ }^{12,25}$

In this work, the potential of multiphoton AF lifetime measurement for label-free imaging of cocultured cells was demonstrated. Future applications include the label-free isolation of iPS cells from feeder cells and other differentiating cells. It helps to optimize the iPS cell culture and provides important information on iPS cell proliferation and differentiation. An optical isolation can, for example, be performed by optical knock out using the same fs NIR laser source. ${ }^{28}$ Also, the label-free separation of iPS cells from their differentiating counterparts within a microfluidic system based on multiphoton AF measurement and multiphoton knock-out may become a valuable tool in iPS research and clinical iPS applications. Femtosecond laser nanoprocessing microscopes have also been used recently to generate iPS cells via optical reprogramming. ${ }^{29}$

\section{Disclosures}

The author declares that there is no conflict of interests.

\section{Acknowledgments}

I wish to thank the German Science Foundation (DFG) for financial support within the "Key Research program 1327/II: Optical Reprogramming of stem cells with sub-15 femtosecond laser pulses." I also would like to thank Dr. Karsten König (Head of the Department) and Ana Batista for their technical support and helpful comments.

\section{References}

1. M. F. Pittenger et al., "Multilineage potential of adult human mesenchymal stem cells," Science 284, 143-147 (1999).

2. Y. Sakaguchi et al., "Comparison of human stem cells derived from various mesenchymal tissues: superiority of synovium as a cell source," Arthritis Rheum. 52, 2521-2529 (2005).

3. J. A. Thomson et al., "Embryonic stem cell lines derived from human blastocysts," Science 282(5391), 1145-1147 (1998).

4. A. Baldwing, "Morality and human embryo research. Introduction to the talking point on morality and human embryo research," EMBO Rep. 10(4), 299-300 (2009).

5. K. Takahashi and S. Yamanaka, "Introduction of pluripotent stem cells from mouse embryonic and adult fibroblast cultures by defined factors," Cell 126, 663-676 (2006)

6. "The Nobel prize in physiology or medicine-2012 press release," Nobel Media AB, www.nobelprize.org/nobel_prizes/medicine/laureates/2012/ press.html (2012).

7. J. Jozefczuk, K. Drews, and J. Adjaye, "Preparation of mouse embryonic fibroblast cells suitable for culturing human embryonic and induced pluripotent stem cells," J. Visualized Exp. 64, e3854 (2012).

8. W. Denk, J. H. Strickler, and W. W. Webb, "Two-photon laser scanning fluorescence microscopy," Science 248(4951), 73-76 (1990).

9. K. König and H. Schneckenburger, "Laser-induced autofluorescence for medical diagnosis," J. Fluoresc. 4(1), 17-40 (1994).

10. I. Bugiel, K. König, and H. Wabnitz, "Investigations of cells by fluorescence laser scanning microscopy with subnanosecond time resolution," Laser Life Sci. 3(1), 47-53 (1989).

11. K. König and A. Uchugonova, "Multiphoton fluorescence lifetime imaging at the dawn of clinical application," in A. Periasamy and
R. M. Clegg, Eds., FLIM Microscopy in Biology and Medicine, pp. 165-187, Taylor \& Francis Group, CRC Press, Boca Raton, London, New York (2009).

12. C. Hu et al., "Energy metabolism plays a critical role in stem cell maintenance and differentiation," Int. J. Mol. Sci. 17, 253 (2016).

13. K. König, A. Uchugonova, and E. Gorjup, "Multiphoton fluorescence lifetime imaging of 3D-stem cell spheroids during differentiation," Microsc. Res. Tech. 74, 9-17 (2011).

14. C. Stringari et al., "Metabolic trajectory of cellular differentiation in small intestine by phasor fluorescence lifetime microscopy of NADH," Sci. Rep. 2, 568 (2012).

15. C. Stringari et al., "Phasor fluorescence lifetime microscopy of free and protein-bound NADH reveals neural stem cell differentiation potential," PLoS One 7(11), e48014 (2012).

16. B. Wright et al., "NADH distribution in live progenitor stem cells by phasor-fluorescence lifetime image microscopy," Biophys. J. 103(1), L07-L09 (2012).

17. A. Meleshina et al., "Probing metabolic states of differentiating stem cells using two-photon FLIM," Sci. Rep. 6, 21853 (2016).

18. S. Huang, A. Heikal, and W. Webb, "Two-photon fluorescence spectroscopy and microscopy of $\mathrm{NAD}(\mathrm{P}) \mathrm{H}$ and flavoprotein," Biophys. J. 82, 2811-2825 (2002).

19. H. G. Breunig, H. Studier, and K. Koenig, "Multiphoton excitation characteristics of cellular fluorophores of human skin in vivo," Opt. Express 18(8), 7857-7871 (2010).

20. G. Weber, "Fluorescence of riboflavin and flavin-adenine dinucleotide," Biochem. J. 47(1), 114-121 (1950).

21. A. J. Visser, "Kinetics of stacking interactions in flavin adenine dinucleotide from time-resolved flavin fluorescence," Photochem. Photobiol. 40(6), 703-706 (1984).

22. A. Uchugonova and K. König, "Two-photon autofluorescence and second-harmonic imaging of adult stem cells," J. Biomed. Opt. 13(5), 054068 (2008).

23. K. P. Quinn et al., "Quantitative metabolic imaging using endogenous fluorescence to detect stem cell differentiation," Sci. Rep. 3, 3432 (2013).

24. R. Datta et al., "Label-free imaging of metabolism and oxidative stress in human induced pluripotent stem cell-derived cardiomyocytes," Biomed. Opt. Exp. 7(5), 1690-1701 (2016).

25. S. Varum et al., "Energy metabolism in human pluripotent stem cells and their differentiating counterparts," PLoS One 6(6), e20914 (2011).

26. J. M. Squirrell et al., "Endogenous fluorescence signatures in living pluripotent stem cells change with loss of potency," PLoS One 7(8), e43708 (2012).

27. A. Uchugonova, A. Batista, and K. König, "Fluorescence lifetime imaging of induced pluripotent stem cells," Proc. SPIE 8948, 89481I (2014).

28. A. Uchugonova et al., "Optical knock out of stem cells with extremely ultrashort femtosecond laser pulses," J. Biophotonics 1(6), 463-469 (2008).

29. A. Uchugonova et al., "Optical reprogramming of human cells in an ultrashort femtosecond laser microfluidic transfection platform," J. Biophotonics 9(9), 942-947 (2016).

Aisada Uchugonova received her PhD degree from Saarland University, Faculty of Medicine in Homburg, Germany, and worked as a postdoctoral researcher at the University of California, San Diego (UCSD). She is the PI of the German Science Foundation funded project on optical reprogramming and embarked on habilitation in the field of femtosecond laser microscopy of stem cells at Saarland University. 